\title{
Geburtshelfer für öko-soziale Innovationen: Konstruktiver Journalismus als Entwicklungskommunikation für westlich-kapitalistische Gesellschaften in der Krise
}

\author{
Uwe Krüger
}

Keywords: Konstruktiver Journalismus, Transformativer Journalismus, Development Journalism, journalistisches Rollenverständnis, Lösungsorientierter Journalismus, Große Transformation, Nachhaltigkeit, Kapitalismus, Berichterstattungsmuster

\section{Abstract}

In westlichen Gesellschaften ist seit Beginn der 2010er Jahre eine kleine Strömung im Journalismus sichtbar geworden, die sich der Berichterstattung über positive Entwicklungen und gelingende Problemlösungen verschrieben hat: Sie nennt sich »Lösungsorientierter « oder "Konstruktiver Journalismus«. Dieser Beitrag diskutiert die theoretische Fundierung des Konstruktiven Journalismus und wie er in Richtung eines "Transformativen Journalismus« weiterentwickelt oder präzisiert werden kann, indem man ihn mit der Idee einer »Großen Transformation « zur Nachhaltigkeit und den Sustainable Development Goals der Vereinten Nationen verknüpft. Abschließend wird das Konzept mit dem des »Development Journalism« verglichen, das seit den 1960er Jahren im Globalen Süden Anwendung findet. Als brauchbares Erbe erscheint dessen Strang, der kritisch und unabhängig über langfristige Entwicklungsprozesse und Innovationen berichtet; zurückgewiesen wird dessen Ausprägung in autoritären Staaten, die Journalist*innen zu PR-Agent*innen der Regierung degradiert. 
Ich danke den Kollegen Holger Pötzsch und Armin Scholl sowie den Studierenden Thilko Gläßgen, Alexandra Hilpert, Maria Ludowika Leidinger, David Muschenich, Kai Remen, Leonie Rottmann, Elisabeth Winkler und Teresa Wolny für ihre konstruktive Kritik des Manuskripts.

Uwe Krüger: Geburtshelfer für öko-soziale Innovationen: Konstruktiver Journalismus als Entwicklungskommunikation für westlich-kapitalistische Gesellschaften in der Krise. In: Nils S. Borchers, Selma Güney, Uwe Krüger und Kerem Schamberger (Hrsg.): Transformation der Medien - Medien der Transformation. Verhandlungen des Netzwerks Kritische Kommunikationswissenschaft. Frankfurt am Main: Westend 2021. DOI: https://doi. org/10.53291/SDTM5470.

Dr. Uwe Krüger I Universität Leipzig | uwe.krueger@uni-leipzig.de

\section{Journalismus: Eine Institution zur Reproduktion des Kapitalismus?}

Eure größte Aufgabe in den kommenden Jahrzehnten besteht darin, innovativ zu sein, aber nicht in Bezug auf Maschinen, sondern in Hinblick auf euch selbst.

(Ismael, in Daniel Quinn: Ismaels Geheimnis, Goldmann 1999, 228)

Kapitalistische Gesellschaften, so sieht es die Kritische Theorie marxistischer Prägung, sind trotz einer krisenanfälligen Wirtschaft und hoher Ungleichheit stabil, weil ihre Mitglieder sie in alltäglichen Tätigkeiten unbewusst und passiv reproduzieren und weil bestimmte Institutionen aktiv für eine Reproduktion sorgen. Zu letzteren gehören staatliche Bürokratie, Polizei und Gerichte, welche die bestehenden Macht- und Herrschaftsstrukturen in letzter Konsequenz mit Zwangsmitteln schützen, sowie das Bildungssystem und die Medien, die auf softere Weise dafür sorgen, dass die Subjektivität der (meisten) Individuen von den herrschenden Normen, Ideen, Werten, Doktrinen und Theorien geprägt wird (vgl. Wright 2017, 378, 388). Nicht nur in der europäischmarxistischen Tradition kritischen Denkens erscheinen die großen Medienhäuser und der darin organisierte redaktionelle Journalismus als Hüter kapitalistischer Wachstums- und Ungleichheitsideologie; auch die nordamerikanischen Spielarten der Critical Political Economy (vgl. Sevignani 2016, 6) sehen sie so. Beispielhaft seien zwei Sätze von Herman und Chomsky $(2002,1)$ über die Massenmedien zitiert: 
It is their function to amuse, entertain, and inform, and to inculcate individuals with the values, beliefs, and codes of behavior that will integrate them into the institutional structures of the larger society. In a world of concentrated wealth and major conflicts of class interest, to fulfil this role requires systematic propaganda.

Europäische Marxist*innen argumentieren, dass Medien die kapitalistische Wirtschaftsweise stützen, indem sie verschiedene Funktionen für das Kapital erfüllen. Knoche $(2002,106)$ nennt derer vier:

- eine Kapitalverwertungsfunktion für die Medienwirtschaft (etwa: Medieneigentümer*innen erzielen mit ihren Produkten Gewinne),

- eine Absatz-, Werbe- und PR-Funktion der Medien für die übrige Wirtschaft (etwa: Werbetreibende Unternehmen machen ihre Produkte oder Marken der Öffentlichkeit bekannt),

- Funktionen der Legitimations- und Herrschaftssicherung sowie der Förderung eines allgemeinen Konsumklimas (etwa: Ideologie wird durch Medieninhalte an die Rezipient*innen vermittelt),

- Funktionen der Regeneration und Qualifizierung des Arbeitsvermögens als Basis für die Kapitalverwertung (etwa: Arbeiter*innen entspannen sich nach einem harten Tag bei Unterhaltungssendungen).

Allerdings: Der in den Medienhäusern betriebene redaktionelle Journalismus als (formal unabhängige) Institution zur Berichterstattung über das aktuelle Geschehen beziehungsweise zur Selbstbeobachtung der Gesellschaft kommt in dieser Sichtweise als Stütze des Kapitalismus ${ }^{1}$ kaum vor. Allenfalls dem Wirtschaftsjournalismus wird eine explizite Funktion für die Kapitalakkumulation zugeschrieben, wenn er Informationen über Märkte und Unternehmen verbreitet und damit Investor*innen und Aktionär*innen bei der Entscheidungsfindung hilft, also eine Rolle in der »Zirkulationssphäre« des Kapitals spielt (vgl. Sevignani

1 Kapitalismus wird hier mit Wright $(2017,78)$ verstanden als »eine bestimmte Art, die Wirtschaftstätigkeit einer Gesellschaft zu organisieren. Er kann anhand zweier Hauptaspekte charakterisiert werden: des Wesens seiner Klassenverhältnisse und seiner zentralen Mechanismen wirtschaftlicher Koordinierung.« (Hervorhebung im Original) Zentrale Merkmale sind Privateigentum an Produktionsmitteln und »Mechanismen des dezentralisierten, freiwilligen Tausches zwischen privat verhandelnden Vertragsparteien « (freier Markt), »wodurch die Preise und Mengen der produzierten Güter und Dienstleistungen bestimmt werden«. Hieraus »geht der charakteristische Wettbewerbstrieb kapitalistischer Betriebe hervor, ihr Streben nach Profit und Kapitalakkumulation« (ebd., 78-79). 
2016, 9). Die nordamerikanischen Kolleg*innen sehen dagegen eine generelle Rolle des Journalismus (zumindest wie er von großen, etablierten Medienhäusern produziert wird) bei der Reproduktion kapitalistischer Macht- und Ungleichheitsstrukturen. Das zentrale Argument hierbei lautet, dass die Journalist*innen großer Medien hauptsächlich den Elitendiskurs abbilden, also die vorhandenen Konflikte zwischen den politischen und wirtschaftlichen Entscheider*innen spiegeln, gleichzeitig aber einen jeweils gegebenen Elitenkonsens (etwa zur geopolitischen Grundorientierung oder makroökonomischen Ordnung) teilen und Dissens von »außen « beziehungsweise »unten « marginalisieren oder delegitimieren. Diese Denkfigur findet sich in verschiedenen Konzepten und Ansätzen, allen voran im Propagandamodell von Herman und Chomsky (2002), aber auch in der Indexing-Hypothese von Bennett, in der Schutzhund-Perspektive von Donohue, Tichenor und Olien oder im Protest-Paradigma von McLeod und Hertog (vgl. Krüger 2019, 46-71).

Die Begründung, warum formal unabhängige Journalist*innen sich mehr oder weniger auf die Abbildung des Elitendiskurses beschränken, fällt jeweils etwas unterschiedlich aus: Einmal ist es die Dominanz offizieller Quellen, bedingt durch ein Bestreben in den Newsrooms, effizient Storys zu produzieren und Druck oder Kritik zu vermeiden; einmal sind es Tiefenstrukturen ökonomischer und politischer Macht beziehungsweise die Verflechtungen der Medienhäuser und ihres Personals mit Institutionen von Staat und Wirtschaft (vgl. ebd., 71-72); aber auch familiärer Hintergrund, Bildungsbiographie und Milieuzugehörigkeit vieler Journalist*innen mögen eine gewisse Eliten-Nähe begünstigen (Krüger 2016, 71-84). Doch selbst in einer wenig gesellschaftskritischen Theorietradition wie der Nachrichtenwertforschung erscheint ein solches Medienverhalten plausibel, denn Nachrichtenfaktoren wie Elite-Personen, Elite-Institutionen, Konflikt, Reichweite und Folgenschwere stellen wichtige Kriterien für journalistische Selektions- und Gewichtungsentscheidungen dar (vgl. Galtung und Ruge 1965; Beiler 2013, 127). Daher dürften Journalist*innen schon qua Gewohnheit und beruflicher Sozialisation den Konflikten im Eliten-Milieu, die potenziell große unmittelbare Auswirkungen auf die Bevölkerung haben, einen hohen Nachrichtenwert zuschreiben. Für die Elitenorientierung großer Medien sind also möglicherweise nicht nur die fünf »Filter« des Propagandamodells ausschlaggebend (Besitzverhältnisse und Medieneigentümer, Werbekunden, hauptsächliche Quellen, Flakfeuer und Ideologie), sondern auch vier »Filter« aus der Nachrichtenwerttheorie, also vier Nachrichtenfaktoren, die sich im Fall von Konflikten im Eliten- 
Milieu stets addieren und so dafür sorgen, dass der Elitendiskurs in der Berichterstattung dominiert und ein jeweiliger Elitenkonsens zu einem Thema quasi automatisch übernommen wird (oder zumindest die Prämissen des Elitendiskurses kaum hinterfragt werden).

Diese Überlegungen führen zu der Annahme, dass (große) Medien beziehungsweise der in ihnen organisierte professionelle Journalismus nicht zwangsläufig und ausschließlich Institutionen zur aktiven Reproduktion einer kapitalistischen Wirtschaftsform darstellen, sondern diese möglicherweise auch passiv und unbewusst reproduzieren. Zumindest gibt es ein mit dem Berufshandeln professioneller Journalist*innen verbundenes Set an redaktionellen Selektionsroutinen und journalistischem Rollenselbstverständnis, das bewusster, begründeter Veränderung zugänglich ist und sich auch gegen die Reproduktion des Kapitalismus wenden kann. Tatsächlich gibt es bereits im journalistischen Feld Akteur*innen, die abweichende Rollenbilder und Routinen ausgeprägt haben, und tatsächlich gibt es nicht den einen Journalismus, sondern verschiedene Journalismen im Sinne von »Berichterstattungsmustern«, die (oft in denselben Zeitungen, Fernsehprogrammen und News-Portalen) nebeneinander herlaufen: Meier (2018, 194-200) nennt neben dem dominanten Nachrichtenparadigma des »Objective Reporting «, das durch seinen Fokus auf die Themen und Positionen etablierter Organisationen am ehesten den Status quo legitimiert und stabilisiert, unter anderem die Muster des »Investigativen«, des »Anwaltschaftlichen « und des »Konstruktiven Journalismus«.

Ziel dieses Beitrags ist es, den Raum für Journalist*innen und Berichterstattungsmuster, die sich gegen die Reproduktion des Kapitalismus wenden oder über ihn hinausgehen und eine (wie auch immer geartete) nachkapitalistische Gesellschaft ${ }^{2}$ befördern wollen, auszuweiten und durch theoretische Begründung abzusichern. Dies erscheint dringlich aufgrund der aktuellen ökologischen und sozialen Vielfachkrise, welche die Stabilität der Gesellschaften und letzten Endes sogar den Fortbestand der menschlichen Art bedroht (Bendell

2 Dabei wird anerkannt, dass kein reales Wirtschaftssystem komplett kapitalistisch (oder sozialistisch oder etatistisch) organisiert ist, sondern stets eine Mischform darstellt. Die Rede vom Kapitalismus meint hier, dass innerhalb des Wirtschaftssystems die kapitalistische Form dominiert; und eine Transformation zu einem nachkapitalistischen Wirtschaftssystem wird nicht als »binärer Übergang von einem System zum anderen begriffen [...], sondern vielmehr als Verschiebung innerhalb der Anordnung der Machtverhältnisse, die eine Mischform ausmachen« (Wright 2017, 488). 
2020; Krüger und Pfeiffer 2020). Diese Krise ist zumindest mitverursacht von systemisch begründeten Wachstums-, Beschleunigungsund Externalisierungszwängen im Kapitalismus (vgl. Dörre et al. 2017; Dörre in diesem Band), weshalb eine »demokratische Postwachstumsgesellschaft « (Krüger und Meyen 2018, 348) erstrebenswert ist.

Der Beitrag folgt der Überlegung, dass die Institutionen zur aktiven gesellschaftlichen Reproduktion »nicht [...] immer wirksam sind « und zudem »Grenzen und Widersprüche « aufweisen, die für gesellschaftliche Emanzipation und Transformation genutzt werden können (Wright 2017, 378). Verschiedene Berichterstattungsmuster und Rollenverständnisse werden hier im Sinne von Widersprüchen und Vielfältigkeit innerhalb des regelgeleiteten Systems »Journalismus « angesehen. ${ }^{3}$ Der Beitrag folgt auch dem von Krüger und Meyen (2018) skizzierten Forschungsprogramm einer »transformativen Kommunikationswissenschaft«. Teil dieses Programms ist es, das Konzept des Konstruktiven Journalismus »so zu formulieren, dass es Nachhaltigkeits- und Gerechtigkeitszielen dient und eine Komponente transnationaler Solidarität enthält, anstatt zu kommerzialisierter Wellness-Berichterstattung für das heimische Wohlstandspublikum zu pervertieren« (ebd., 353). Im Folgenden wird daher der Konstruktive Journalismus mit der Idee einer "Großen Transformation « zur Nachhaltigkeit und dem aus dem Globalen Süden stammenden Konzept des Entwicklungsjournalismus (»Development Journalism«) zusammengebracht, um daraus Folgerungen für das Rollenverständnis und die Selektionsroutinen eines »Transformativen Journalismus« im Globalen Norden zu ziehen.

\section{Der Trend des Konstruktiven Journalismus}

Unter dem Oberbegriff »Konstruktiver Journalismus« werden ungefähr seit den letzten zehn Jahren im überwiegend europäischen und amerikanischen Raum journalistische Ansätze diskutiert, die sich dem vorherrschenden »negativity bias« in den Medien entgegenstellen und unter Einbezug

3 Journalismus wird hier im Sinne des Neo-Institutionalismus als Institution angesehen, da er als dauerhaft angelegtes Regelsystem sowohl soziales Handeln begrenzt und ermöglicht als auch auf seine Umwelt im normierenden, regulierenden, konstitutiven und repräsentativen Sinne einwirkt (vgl. Donges 2006, 568-569). Journalismus ist dann »eine Erwartungsstruktur, die durch soziale Praktiken über große Spannen von Raum [...] und Zeit [...] hinweg reproduziert wird« (Buschow 2012, 36). 
von Erkenntnissen der positiven Psychologie eine vermehrt konstruktive, lösungsorientierte Berichterstattung von Journalist*innen einfordern.

So umreißt Schmidt $(2017,15)$ den Trend, der hier von Interesse ist und der sich in den vergangenen Jahren etwa in der Gründung des Solutions Journalism Network in New York (2013), des Constructive Institute an der Universität Aarhus (2017) und des Constructive Journalism Network (2017) äußerte. Seit 2013 veranstaltet das Pariser Medien-Startup Sparknews jährlich einen weltweiten »Impact Journalism Day«, an dem Zeitungen aus aller Welt untereinander lösungsorientierte Artikel austauschen und gesammelt drucken. Spezialisierte Zeitungen, Zeitschriften und Onlineportale wurden gegründet, beispielsweise Positive News (1994) in Großbritannien, Yes! (1996) in den USA sowie enorm (2010), oya (2010), Perspective Daily (2015), Kater Demos (2015), transform (2015) und andere in Deutschland. Rundfunkanstalten führten lösungsorientierte Rubriken und Sendereihen ein, wie NDR Info »Perspektiven« (2016), das ZDF die Doku-Reihe »Plan B« (2017) und tagesschau.de den »Lösungsfinder« (2019). Außerdem bringen Zeitungen vermehrt Artikelserien und Sonderausgaben mit Good News (Beispiele in Kramp und Weichert 2020; Meier 2018; Krüger und Gassner 2014). Breit rezipiert wurden in der Medienbranche einige Bücher zum Thema von Vordenker*innen und Protagonist*innen der Szene (Haagerup 2015; Gyldenstedt 2015; Urner 2019).

Der Trend ist weiterhin ablesbar an der Veranstaltung spezialisierter Konferenzen (»Global Constructive Journalism Conference« seit 2017 an der Universität Aarhus, »Constructive Journalism Day« seit 2018 von Hamburg Media School und NDR), der Auslobung eines Preises (»Global Constructive Journalism Award«) und der Aufnahme des Themas in die Curricula von Journalist*innenschulen und Hochschulen. Konstruktiver Journalismus wird zudem von Stiftungen gefördert: in Deutschland von der Noah Foundation und der Schöpflin-Stiftung, die seit 2017 »Schöpflin-Stipendien für lösungsorientierten Journalismus« vergeben, von der in Wien ansässigen UNGSII Foundation (United Nations Global Sustainability Index Institute), die seit 2017 den »Global Constructive Journalism Award « finanziert, und von Dutzenden USStiftungen, die das Solutions Journalism Network unterstützen (darunter Knight Foundation, Bill \& Melinda Gates Foundation und Rockefeller Foundation). ${ }^{4}$

4 Die Geldgeber des SJN finden sich unter: https://www.solutionsjournalism. 
Aber mit Hilfe welcher theoretischen Konzepte kann Konstruktiver Journalismus nun genauer gefasst werden? Meier (2018, S. 7-8) sieht ihn als ein »alternatives Berichterstattungsmuster«, das den klassischen »objektiven Journalismus« (der einen Fokus auf offizielle Standpunkte und von mächtigen Institutionen inszenierten Ereignisse legt) ergänzt und dabei in der Tradition des »Civic « oder »Public Journalism«steht, welcher auf lokaler Ebene Lösungen recherchieren beziehungsweise durch die Organisation entsprechender Foren und demokratischer Prozesse selbst anstoßen will. Konstruktiver Journalismus zeichnet sich laut Meier (ebd.) dadurch aus, dass er einerseits nicht ereignisfixiert ist, sondern langfristige Prozesse in den Blick nimmt, und andererseits nicht nur soziale Probleme beschreibt, sondern auch Debatten über mögliche Lösungen anschieben will. Diese Gegenüberstellung von Problem(-orientierter Berichterstattung) und Lösung(-sorientierter Berichterstattung), die auch in Statements von Praktiker*innen des Feldes häufig anzutreffen ist (vgl. Kramp und Weichert 2020), verweist auf die Möglichkeit, die Idee des Konstruktiven Journalismus an das kommunikationswissenschaftliche Konzept des »Framing « anzuschließen (vgl. Krüger 2016, 107). Ein Frame - also ein kognitiver Rahmen beziehungsweise ein Deutungsmuster, mit dem Menschen einen bestimmten Ausschnitt aus der Realität fokussieren und damit Komplexität reduzieren - besteht nach der klassischen Definition von Entman (1993) aus vier Elementen: 1) Thema oder Problem, 2) Ursachenzuschreibung, 3) Bewertung und 4) Lösung oder Handlungsempfehlung. Entsprechend schreibt McIntyre (2019, 30): "In solutions journalism, the frame is such that the solution part of the story is highlighted, whereas traditional news stories often make the problem part of the story more salient."

Zugleich macht McIntyre gemeinsam mit Gyldenstedt die Verbindung zu einem anderen theoretischen Konstrukt stark: der Positiven Psychologie. Dabei handelt es sich um eine Strömung innerhalb der Psychologie und Psychotherapie, welche die Ressourcen von Patient*innen anstelle von deren Defiziten in den Mittelpunkt stellt, etwa um Depressionen zu

org/who-we-are/funders. Die Unterstützung durch die philanthropischen Arme von Superreichen und Konzernen wirft freilich die Frage auf, ob Konstruktive Journalist*innen dann die Ideologie des »Philanthrokapitalismus« übernehmen und mit Themenwahl und Framing den Status quo jenes globalen Kapitalismus unterstützen, aus dem die Geldgeber ihren Reichtum bezogen haben (vgl. Scott et al. 2017, 168) - obwohl sie angesichts der multiplen Krisen unserer Zeit eigentlich die Systemfrage stellen müssten. 
heilen, die aufgrund von »erlernter Hilflosigkeit« auftreten. Während klassische problemzentrierte Berichterstattung den Rezipient*innen ein Gefühl von Hilflosigkeit vermittele und zu Apathie und Zynismus führe, wende Konstruktiver Journalismus Techniken der Positiven Psychologie an, »in an effort to create productive and engaging coverage, while holding true to journalism's core functions « (McIntyre und Gyldenstedt 2017, 23; vgl. Beiler und Krüger 2018, 176-178). Mit der Entscheidung für dieses Konzept aus der Individualpsychologie betonen McIntyre und Gyldenstedt die Mikro-Ebene des Individuums als Bezugspunkt des Konstruktiven Journalismus und begründen die Notwendigkeit des Berichterstattungsmusters vorrangig mit dem persönlichen Wohlbefinden der Nutzer*innen, welches es zu erhalten oder herzustellen gelte.

Allerdings: Historisch gesehen hatten die Vorläufer der heutigen Strömung mit Positiver Psychologie nichts im Sinn. Dem Publizisten und Zukunftswerkstätten-Erfinder Robert Jungk, der Ende der 1940er Jahre mit dem »Good News Bulletin« den Aufschlag machte (vgl. Krüger 2016, 101-103), und dem Nachrichtenwert- und Friedensforscher Johan Galtung, der lösungsorientierten »Friedensjournalismus« statt gewaltorientierten »Kriegsjournalismus « forderte, ging es eher um gesellschaftlichen Fortschritt auf der Makro-Ebene: Mit einer bewussten Änderung eingeschliffener redaktioneller Selektions- und Gewichtungsroutinen (Stichwort: Nachrichtenwerttheorie) sollte Journalismus gewaltfreie Lösungsansätze, öko-soziale Innovationen und emanzipatorische Bewegungen aus der Zivilgesellschaft öffentlich sichtbar machen und fördern.

Nun kann die derzeitige (kleine) Strömung des Konstruktiven Journalismus in der Medienbranche zunächst als Antwort auf die Krise der Medienbranche selbst gesehen werden. Diese Krise betrifft die Aspekte Finanzierung, Reichweite, Glaubwürdigkeit und Status in der Gesellschaft und hängt mit dem aktuellen Strukturwandel der Öffentlichkeit im Zuge von Digitalisierung und Globalisierung zusammen (vgl. Krüger 2018; Jarren 2015). Wenn etablierte Medienhäuser sich in Konstruktivem Journalismus versuchen, werden sie wohl nicht nur einen Nutzen auf der individuellen Mikro-Ebene oder gesamtgesellschaftlichen Fortschritt auf der Makro-Ebene anstreben, sondern auch und vor allem einen Nutzen auf der Meso-Ebene - also für ihre eigene Organisation (vgl. Krüger 2016, 98-101). Demgegenüber ist die Sehnsucht vieler Journalist*innen und Rezipient*innen nach mehr lösungsorientierter Berichterstattung ${ }^{5}$ wohl auch Reaktion auf eine gesamtgesellschaftli-

5 Eine solche Sehnsucht zeigt sich in Statements Konstruktiver Journalist*innen 
che Krisenhaftigkeit, die mit einer grundstürzenden »Metamorphose der Welt« (Beck 2017) in Richtung Kosmopolitismus, mit globalen Bedrohungen wie dem Klimawandel und unbewältigten »weltinnenpolitischen « Gerechtigkeits- und Ungleichheitsproblemen zu tun hat (die beispielsweise einen hohen Migrationsdruck nach sich ziehen). Sie ist sicher auch Reaktion auf eine wahrgenommene systemische Starre beziehungsweise Alternativ- und Utopielosigkeit innerhalb westlich-kapitalistischer Wachstumsgesellschaften, die ihre Versprechen auf ständig steigenden Wohlstand nicht mehr erfüllen können und deren mentale Infrastrukturen und grundlegende Fortschrittsnarrative erodieren (vgl. Welzer 2013).

So sieht sich zumindest ein Teil der Akteur*innen im Feld des Konstruktiven Journalismus als »Geburtshelferin ökosozialer Innovationen« (Ute Scheub), als »Change Agent für die Große Transformation« (Manfred Ronzheimer) oder als "Sterbebegleiter für ein abgewirtschaftetes System und Geburtshelfer für eine neue Kultur « (Geseko von Lüpke; alle zitiert nach Krüger und Gassner 2014, 25; Krüger 2016, 100-101). Diesen Teil des Feldes bezeichne ich als systemkritische »Jungk-Fraktion«, die neben einer systemimmanenten, kapitalismusfreundlichen »Ashoka-Fraktion« existiert (benannt nach der Organisation Ashoka zur Förderung des Sozialen Unternehmertums, in der einige Akteur*innen im Feld vernetzt sind, vgl. Krüger 2016, 105-106).

Während letztere sich politisch neutral gibt und epistemologisch die Position vertritt, dass der Journalismus erst mit einem konstruktiven Ansatz sein Versprechen auf Objektivität einlösen kann (»Mainstream media has a bias for bad news « ${ }^{6}$ ), bekennt sich die »Jungk-Fraktion « zu Subjektivität und Normativität und möchte einen grundlegenden, mehr oder weniger zielgerichteten Wandel in Richtung Postwachstumsökonomie mit publizistischen Mitteln unterstützen. In jenem Teil des Feldes könnte man nicht (nur) von Konstruktivem, sondern sogar von "Transformativem Journalismus« sprechen. Es fragt sich nur: Welche Art Transformation wird genau angestrebt? Im Sinne welcher Werte und politischen Wunschvorstellungen soll sich die Gesellschaft grundlegend verändern und zu welchen Mitteln dürfen und sollen Journa-

(etwa in Krüger und Gasser 2014; Kramp und Weichert 2020) und in repräsentativen Bevölkerungsumfragen (Loosen, Reimer und Hölig 2020; weitere Umfragen zitiert in Beiler und Krüger 2018, 169-170).

6 Slogan des Solutions Journalism Network bei dessen Start 2012: https://web. archive.org/web/20130209025903/http://solutionsjournalism.org/. 
list*innen dafür greifen? Ist die Konstruktive beziehungsweise Transformative Journalistin dann noch Beobachterin und Berichterstatterin oder eher Aktivistin, PR-Frau und Politikerin? In welcher Beziehung steht sie dann zu (anderen) Aktivist*innen und relevanten Akteur*innen wie Eliten aus Politik und Wirtschaft? Verletzt ein Engagement für eine gezielte Transformation wichtige Kriterien journalistischer Qualität, wie Unabhängigkeit, Unparteilichkeit, Kritik, Objektivität, Ausgewogenheit und Neutralität? Diese Fragen sollen im Folgenden zumindest ansatzweise beantwortet werden.

\section{Die »Große Transformation « zur Nachhaltigkeit}

Polanyi (1957) war der erste, der von dem Begriff einer »Großen Transformation« Gebrauch machte. Sie bezeichnet bei ihm den Übergang von der feudalen Agrargesellschaft zur kapitalistischen Industrie- und Marktgesellschaft, der sich vor allem im 19. Jahrhundert ereignete und neben der Struktur des Wirtschaftens auch soziale und kulturelle Normen und Werte fundamental veränderte. Seitdem hat der Kapitalismus für einen historisch beispiellosen Anstieg der Produktivität und des Wohlstands gesorgt, allerdings auch für gravierende ökologische Probleme, materielle Ungleichheiten und daraus folgende Defizite in sozialer und politischer Gerechtigkeit (vgl. Krüger und Meyen 2018, 348-350; Wright 2017, 53). Der Wissenschaftliche Beirat der Bundesregierung Globale Umweltveränderungen (WBGU) fordert daher in seinem Hauptgutachten »Welt im Wandel - Gesellschaftsvertrag für eine Große Transformation« eine neuerliche Umwälzung von Werten, Normen und Institutionen: „Es geht um einen neuen Weltgesellschaftsvertrag für eine klimaverträgliche und nachhaltige Weltwirtschaftsordnung «, schreibt etwa der WBGU (2011, 1-2, Hervorhebung im Original). Er verlangt weiterhin »die Schaffung eines nachhaltigen Ordnungsrahmens, der dafür sorgt, dass Wohlstand, Demokratie und Sicherheit mit Blick auf die natürlichen Grenzen des Erdsystems gestaltet « (ebd., 1) werden. Auch andere Forscher*innengruppen weltweit vertreten diese Idee, so das Sustainability Transitions Research Network (STRN) und das Great Transition Network (GTN) mit dem Tellus Institute in Cambridge, Massachusetts (vgl. Göpel 2016).

Das gesellschaftliche Kernprinzip, das die Richtung der erwünschten neuen Transformation angibt, ist »Nachhaltigkeit (englisch: sustainability). Damit gemeint ist, dass »die Bedürfnisse der gegenwärtigen 
Generationen befriedigt werden, ohne dass die Bedürfnisse zukünftiger Generationen beeinträchtigt werden « (Kannengießer 2020, 12). Der Begriff besitzt »normativ-politischen Charakter« (Neverla 2020, 335) und "hat sich etabliert als Gegenentwurf zum ressourcenverschlingenden Leben der Spätmoderne, als kritische Gegenposition zur herrschenden sozialen Logik « (ebd., 336). Allgemein akzeptiert ist die Vorstellung, dass Nachhaltigkeit eine ökologische, eine soziale und eine wirtschaftliche Dimension hat, umstritten ist hingegen, ob diese gleichwertig nebeneinander stehen (in einem »Drei-Säulen-Modell«) oder hierarchisch ineinander eingebettet sind (in einem »Vorrang-Modell«) - die Wirtschaft in der Gesellschaft und die Gesellschaft wiederum in der Umwelt, so dass ökologische Nachhaltigkeit Vorrang vor sozialer und soziale Nachhaltigkeit Vorrang vor wirtschaftlicher hat (vgl. Göpel 2016, 87-88).

Nachhaltigkeit ist ein globales Leitbild geworden, spätestens mit der Verabschiedung der »Agenda 2030 für nachhaltige Entwicklung « durch die Vereinten Nationen im Jahr 2015: Sie definiert 17 Sustainable Development Goals (SDGs) mit 169 konkreten Unterzielen (Abb. 1; vgl. Clauß in diesem Band). Auch die Bundesregierung bekennt sich in ihrer »Deutschen Nachhaltigkeitsstrategie« zu Generationengerechtigkeit, dem Schutz der natürlichen Lebensgrundlagen und sozialer Verantwortung auch gegenüber ärmeren Ländern als Maßstäbe des Regierungshandelns und spricht von einer "notwendigen wirtschaftlichen und gesellschaftlichen Transformation « (Bundesregierung 2018, 11), ohne freilich die Dysfunktionalitäten und Machtstrukturen des modernen globalen Kapitalismus zu problematisieren und einen »radikalen, inkrementellen Wandel « des Wirtschaftssystems zu fordern, wie es ehemalige Angehörige und Mitarbeiter*innen ihres Beirats WBGU tun (Schneidewind 2018, 65-106; Göpel 2016). Wichtig ist jedoch der Perspektiv- und Paradigmenwechsel, der mit der Definition und Operationalisierung von »nachhaltiger Entwicklung « durch die Vereinten Nationen einhergeht und alle Länder - auch Deutschland - in die Pflicht nimmt: Entwicklung soll nicht mehr nur eine Aufgabe für "Entwicklungsländer « sein und vor allem wirtschaftliches Wachstum nach westlichem Vorbild im Sinne einer »nachholenden Entwicklung « bezeichnen. Legt man den Maßstab der 17 SDGs an (darunter Herstellung von Geschlechtergerechtigkeit, Beendigung von Armut, Verringerung von Ungleichheit sowie Gewährleistung nachhaltiger Konsum- und Produktionsmuster), so sind auch ökonomisch erfolgreiche Länder im Zentrum des Weltwirtschaftssystems in vielerlei Hinsicht »Entwicklungsländer« (die durchaus auch in bestimmten Punkten von 
den traditionell so betitelten Staaten lernen können, vgl. Schneidewind 2018, 119).

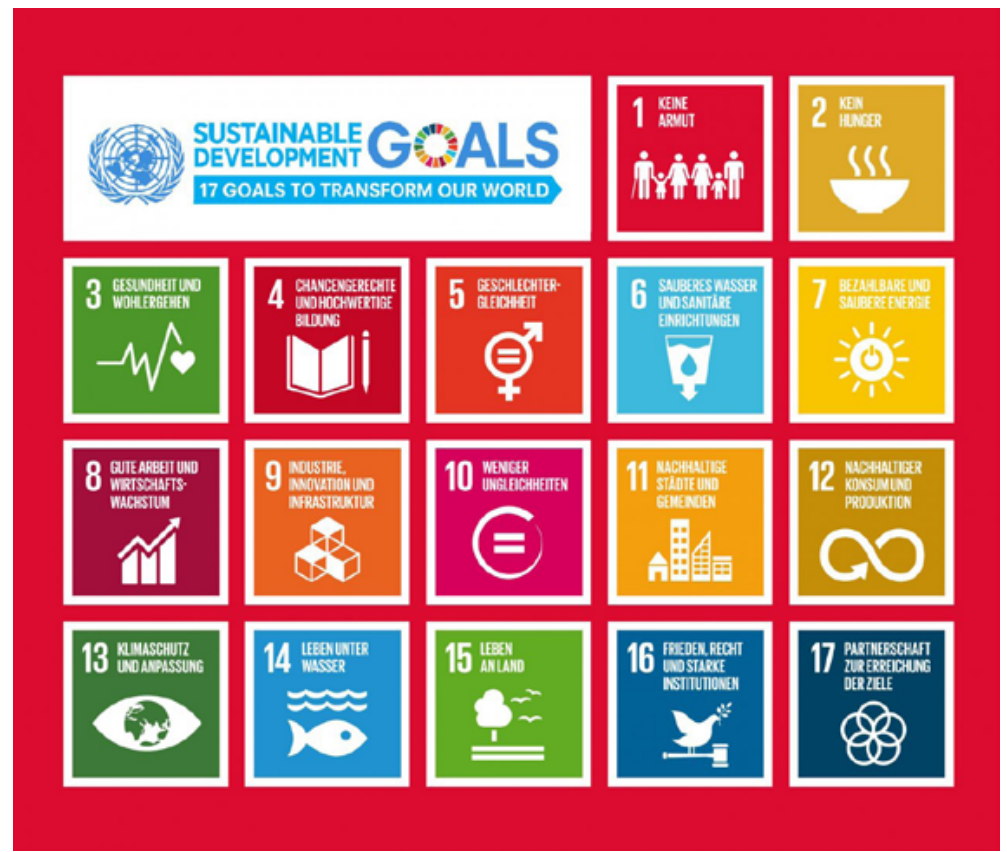

Abb. 1: Die Ziele für nachhaltige Entwicklung der Vereinten Nationen, verabschiedet 2015 (Quelle: UN, CC-BY-SA 3.0, https://www.unesco.de/ bildung/agenda-bildung-2030/bildung-und-die-sdgs).

Das revolutionäre Moment des Begriffs »Nachhaltigkeit« darf also trotz dessen inflationärer Verwendung in Medien, Wirtschaft und Politik nicht übersehen werden. Schneidewind (2018, 23, 25) stellt dieses Moment eindrucksvoll heraus, wenn er die Idee der nachhaltigen Entwicklung als ein »kulturelles Projekt« und ein »umfassendes Zivilisationsprojekt« bezeichnet sowie als eine »moralische Revolution«, die Ähnlichkeiten mit früheren moralischen Revolutionen wie der Einführung der Demokratie, der Abschaffung der Sklaverei und der Einführung des Frauenwahlrechts hat:

Das Konzept der Nachhaltigen Entwicklung liegt [...] genau auf dieser zivilisatorischen Entwicklungslinie: Es ist eine systematische Erweiterung der Idee der Menschenrechte, indem sie allen Menschen auf diesem Planeten so- 
wie auch zukünftigen Generationen die gleichen Entwicklungsmöglichkeiten eröffnen möchte. Es beschreibt ein wachsendes Verständnis des Respekts gegenüber anderen Menschen, global und intergenerationell. (ebd., 26)

Sollte diese moralische Revolution eines Tages vollendet und »die Würde und die Entfaltungsmöglichkeiten von Menschen überall auf dieser Welt heute und in Zukunft Kompass für gesellschaftliches, politisches und ökonomisches Handeln« (ebd., 23) geworden sein, wird die Menschheit sich übrigens - ähnlich wie bei früheren moralischen Revolutionen - fragen, wie die alte Praxis jemals bestehen konnte. Rückblickend bleibt nur Kopfschütteln: „Was haben wir da nur gedacht? Wie konnten wir das all die Jahre tun?« (Appiah 2011, zitiert in Schneidewind 2018, 28)

\section{Transformativer Journalismus und sein Verhältnis zum »Development Journalism«}

Zur Unterstützung der »Großen Transformation« fordert der WBGU (2011) eine »Transformative Wissenschaft« und eine »Transformative Bildung «, die transformationsrelevantes Wissen generieren und vermitteln sowie auch Veränderungen in die gewünschte Richtung anstoßen sollen. Er erkennt auch die Bedeutung von Kommunikationstechnologien und öffentlicher Kommunikation für den angestrebten gesellschaftlichen Such- und Aushandlungsprozess an, der demokratisch und partizipativ anzulegen sei. Genaueres zu öffentlichkeitsbezogenen Fragen - etwa zur Rolle von Strategischer Kommunikation und Journalismus - ist nicht zu finden. An anderer Stelle erwähnt das ehemalige WBGU-Mitglied Schneidewind $(2018,357)$ im Zusammenhang mit dem Konstruktiven Journalismus einen »Transformativen Journalismus«, der »analog zu einer Transformativen Wissenschaft [...] Pionierinnen und Initiativen des Wandels ermutigt, orientiert, informiert und vernetzt«. In ähnlicher Weise verwenden Ronzheimer (2013) sowie Borner et al. (2019) den Begriff, ebenfalls ohne ihn kommunikationswissenschaftlich beziehungsweise journalismustheoretisch zu fundieren.

Zunächst soll hier eine Arbeitsdefinition vorgestellt werden, welche die Analogie zur Transformativen Wissenschaft aufnimmt, aber den Gegenstand weiter spezifiziert:

Transformativer Journalismus ist eine Form der Berichterstattung, die Öffentlichkeit herstellt für Akteur*innen, Prozesse und Strukturen, die eine »Große 
Transformation» zur Nachhaltigkeit begünstigen, um sie durch Sichtbarkeit zu stärken und ihre weitere Verbreitung und Entwicklung zu ermöglichen. Er ist normativ verankert in der Nachhaltigkeitsdebatte und den SDGs; diese Verankerung versteckt er nicht, sondern legt sie offen und reflektiert sie kritisch. Grundlegende Werte für den Transformativen Journalismus sind die Schonung ökologischer Ressourcen und die Einhaltung planetarer Grenzen, soziale Gerechtigkeit, transnationale Solidarität und Demokratie. Seine typischen Themen drehen sich um die »sieben Arenen « der Großen Transformation nach Schneidewind (2018, 14): Wohlstands- und Konsumwende, Energiewende, Ressourcenwende, Mobilitätswende, Ernährungswende, Urbane Wende und Industrielle Wende.

Von Strategischer Kommunikation und Öffentlichkeitsarbeit grenzt sich der Transformative Journalismus dadurch ab, dass er institutionell und mental unabhängig von den Akteur"innen des Wandels agiert und daher auch mit nüchternem Blick deren Fehler, Misserfolge und Missstände sieht und offenlegt. Trotz seiner Wertegebundenheit erfüllt er also zentrale journalistische Qualitätskriterien wie Unabhängigkeit, Kritik und Objektivität. ${ }^{7}$

Transformativer Journalismus im Dienst einer »nachhaltigen Entwicklung« lässt sich als Teilmenge zweier größer gezogener Konstrukte begreifen:

1. von Nachhaltigkeitskommunikation, einem »Verständigungsprozess, in dem es um eine zukunftsgesicherte gesellschaftliche Entwicklung geht, in deren Mittelpunkt das Leitbild einer Nachhaltigen Entwicklung steht« (Michelsen 2007, 27).

2. von Entwicklungskommunikation oder Communication for Social Change, einer »Sammelbezeichnung für die vielfältigen Formen von Kommunikationsstrategien [...], die gesellschaftliche Veränderungspotenziale sondieren oder aktivieren« (Hamidi und Mielke Möglich 2019, 5).

7 Viele Vertreter*innen des Konstruktiven Journalismus lehnen das Qualitätskriterium »Objektivität« als Schimäre oder Berufsideologie ab. Hier wird das Kriterium jedoch befürwortet und eingefordert, verstanden als Abstrahierung von einer rein persönlich-individuellen Subjekt-Perspektive und »schrittweises Erweitern der Perspektive in Richtung eines (nie zu erreichenden) >Blicks von nirgendwo« (Schultz 2020). Mit einer Kopplung des Transformativen Journalismus an bestimmte Werte und normative Wunschvorstellungen erscheint eine so verstandene Objektivität vollauf vereinbar, da sie keine (Wert-)Neutralität voraussetzt. 
Auf der Ebene des Journalismus als spezifischer Kommunikationspraxis beziehungsweise -institution sind wiederum zwei semantisch ähnliche Begriffe in der Diskussion:

1. der Nachhaltigkeitsjournalismus, eine junge Begriffsprägung von Wissenschaftler*innen der Leuphana Universität Lüneburg, welche ein Studienprogramm »Nachhaltigkeit und Journalismus« anbietet; der Begriff "fasst journalistische Aktivitäten zusammen, die einen Beitrag zur Debatte des Konzepts einer nachhaltigen Entwicklung leisten beziehungsweise sich darauf beziehen« (Michelsen und Fischer 2016, 10). ${ }^{8}$

2. der Entwicklungsjournalismus (Development Journalism), ein Begriff aus dem Globalen Süden mit über 50-jähriger Geschichte, der für eine Diskussion über Qualitätskriterien eines Transformativen Journalismus besonders interessant ist.

Wenn wir Transformativen Journalismus als eine Art kommunikative Entwicklungshilfe für entwickelte (westlich-kapitalistische) Gesellschaften in der Krise verstehen, liegt folgende Frage nahe: Kann der Globale Norden - der seine Entwicklungsgeschichte als Königsweg, seine Werte und Institutionen als Muster und Vorbild für eine erfolgreiche Entwicklung des Globalen Südens ansah, heute aber selbst in einer Sackgasse steckt und sich grundlegend wandeln muss - eventuell hier von Asien, Afrika sowie Süd- und Mittelamerika ${ }^{9}$ lernen? Was kann er vom Konzept des Development Journalism übernehmen, was sollte er zurückweisen?

Das Konzept des Development Journalism entstand Ende der 1960er Jahre im Zuge eines Workshops für Wirtschaftsjournalist*innen, den der britische Journalist und Asien-Kenner Alan Chalkley 1968 in Manila (Philippinen) leitete. In seinem »Manual of Development Journalism« wird deutlich, dass es ihm um kritischen, akkuraten Wirtschafts-

8 Die Autoren sehen den Nachhaltigkeitsjournalismus wiederum in semantischer Nähe zu »Umweltjournalismus«, »Klimajournalismus«, »Grünem Journalismus« (vgl. Schäfer und Lorenz in diesem Band) und »Transformationsjournalismus« nach Ronzheimer (2013).

9 Einschränkend muss gesagt werden, dass die Grenze zwischen Globalem Norden und Globalem Süden nicht strikt anhand von Kontinenten verläuft. Beispielsweise zählt Japan, obwohl ein asiatischer Staat, nicht zum Globalen Süden, während arme europäische Länder wie Albanien oder Moldawien nicht unter den Begriff des Globalen Nordens fallen. 
journalismus unter den besonderen Umständen von weit verbreiteter Armut und mangelhaften amtlichen Wirtschaftsstatistiken ging. Entwicklungsjournalist*innen haben, so Chalkley, drei Aufgaben: 1) die Rezipient*innen zu informieren, also ihnen die Fakten zu liefern, 2) die Fakten zu interpretieren und in den richtigen Rahmen zu setzen und 3) den Rezipient*innen die Augen für mögliche Lösungen zu öffnen, um den Teufelskreis der Armut zu durchbrechen (Chalkley 1969, 3). Ein wichtiges Anliegen war ihm, dass über Wirtschaft auf eine Weise berichtet wird, die nicht (nur) für Eliten und Entscheider*innen, sondern auch für einfache Bürger*innen verständlich ist:

Tell the housewife $[\ldots]$ about the things that affect her little home economy and home budget. [...] Tell the »faceless" people, with no titles, no names, perhaps no jobs, no money. Bring them into the circle, give them a role to play. (ebd., 53)

Neben der Lösungsorientierung war also auch Ermächtigung zur Teilhabe die Grundidee; es ging um »furthering the emancipation of such deprived groups as the urban poor, the rural people, women and so on and helping them actively to participate in the political process, that is actively influence their destinies« (Quebral 1975; zitiert nach Xu 2009, 359).

Chalkleys Idee machte in den 1970er und 1980er Jahren vor allem in Asien und Afrika Karriere. Für die Staaten, die gerade ihre politische Unabhängigkeit erlangt hatten, schien es attraktiv, nicht das liberaldemokratische Journalismusmodell ihrer westlichen Kolonisatoren zu übernehmen, sondern ein neues Konzept zu finden, das ihren besonderen lokalen Bedingungen (wie politischer Instabilität, ethnischer Vielfalt und prekärer Wirtschaftslage) und kulturellen Werten (wie Betonung von Harmonie statt Konflikt, Vorrang von Gruppeninteressen vor individueller Autonomie und Respekt vor Autoritäten) entsprach. Ideologisch eng verknüpft mit Bestrebungen einer »De-Westernization« und der Bemühung um eine »New World Information and Communication Order« (NWICO) im Rahmen der UNESCO, entstanden oftmals mit staatlicher Förderung Strukturen der journalistischen Ausbildung, die das Rollenbild des Development Journalism propagierten. Dabei entwickelte sich die Idee durchaus widersprüchlich weiter. Laut Xu $(2009,358)$ enthält sie fünf Schlüsselkomponenten:

1. Berichterstattung über Entwicklungsprojekte und die Differenz zwischen deren proklamierten Zielen und den erreichten Ergeb- 
nissen sowie zwischen den behaupteten und realen Auswirkungen auf die Menschen,

2. Schwerpunkt auf langfristigen Entwicklungsprozessen statt auf aktuellen Ereignissen,

3. Unabhängigkeit von und konstruktive Kritik an der Regierung,

4. Schwerpunkt auf wirtschaftlicher und sozialer Entwicklung bei gleichzeitiger konstruktiver Zusammenarbeit mit der Regierung beim Nation-Building,

5. Ermächtigung von Normalbürger*innen, ihr Leben und ihre Gemeinschaften zu verbessern.

Von Forscher*innen ist das Konzept kritisiert worden, weil es in einigen Ländern von autoritär Regierenden dazu missbraucht wurde, um Konformität von den Medien einzufordern - am Ende diente die Idee also oftmals dem Machterhalt der Elite und nicht der Emanzipation der Deprivierten (Xu 2009; Waisbord 2010; Cenite et al. 2008; Hanusch und Uppal 2015). In seinem kritischen Review der Idee konstatiert Waisbord (2010, 150), dass das Konzept kein einheitliches Set an theoretischen Prinzipien aufweist, weil es die Wünsche und Erwartungen von vielen unterschiedlichen Akteur*innen im Globalen Süden ausdrückt; es werde lediglich zusammengehalten in der kategorischen Ablehnung des liberalen Modells der Presse beziehungsweise eines jeglichen westlichen Kommunikationsparadigmas.

Waisbord spitzt die widersprüchliche Lage in Theorie und Praxis des Development Journalism zu zwei unterschiedlichen Ansätzen zu: Der »communitarian approach « fokussiere auf Nicht-Eliten, eigentumslose und arme Menschen sowie deren Lebensumstände, biete Perspektiven und Lösungen, beobachte kritisch die Regierungspolitik sowie Entwicklungsprogramme und fördere Bürgerbeteiligung und Emanzipation. Im Gegensatz dazu sehe der "statist approach « den Journalismus als Instrument des Nation-Building und als Unterstützer von Regierungsprogrammen, denn Entwicklungsziele hätten Priorität vor Konflikten und Meinungsverschiedenheiten; junge und fragile Staaten könnten sich keine freie und oppositionelle Presse leisten.

An dieser Zuspitzung wird klar, was Transformativer Journalismus im Globalen Norden von diesem Modell aus dem Globalen Süden übernehmen kann - Fokus auf langfristige Prozesse, Wissenstransfer über öko-soziale Innovationen und Beobachtung von Entwicklungsprojekten - und wo es gefährlich wird: Die in langen, opferreichen Kämpfen erstrittene Pressefreiheit und Unabhängigkeit von Machthaber*innen 
sollte ein Transformativer Journalismus keinesfalls aufgeben; zu einem PR- und Verlautbarungsorgan von politischen, wirtschaftlichen oder zivilgesellschaftlichen Protagonist*innen einer Nachhaltigkeitsrevolution darf er sich - bei aller Sympathie und Übereinstimmung in den grundlegenden Werten - nicht degradieren lassen. Da aller Macht die Gefahr von Machtmissbrauch innewohnt, bleibt die Watchdog-Funktion selbst für Transformative Journalist*innen relevant. Denn politische Akteur*innen - seien es die herrschenden Eliten oder aufstrebende Gegen-Eliten - benutzen die Wahrheit im Zweifel instrumentell zur Erreichung ihrer politischen Ziele. Die Aufgabe von Journalist*innen ist es deshalb, einen eigenständigen »öffentlichen Wahrheitsdiskurs« zu führen, der über den »Herrschaftsdiskurs« hinausgeht und diesen permanent kritisch hinterfragt (vgl. Pöttker 2003, 150).

\section{$5 \quad$ Fazit und Ausblick}

Die Presse könne »mit einem Gerüst verglichen werden, das um ein im Bau befindliches Gebäude errichtet wird; es zeigt die Umrisse des Gebäudes an, erleichtert den Verkehr zwischen den einzelnen Bauarbeitern, hilft ihnen, die Arbeit zu verteilen und die durch die organisierte Arbeit erzielten gemeinsamen Resultate zu überblicken « (zitiert nach Autorenkollektiv 1981, 72). Diese Baugerüst-Metapher scheint zur Idee des Konstruktiven Journalismus, der zu gesellschaftlichem Fortschritt beitragen will, gut zu passen - und vielleicht noch besser zu einem Transformativen Journalismus, der eine Nachhaltigkeitsrevolution befördern, also gezielt an der »Konstruktion« einer besseren, gerechteren, nachkapitalistischen Welt mitwirken will. Das Zitat stammt auch von einem ausgewiesenen Antikapitalisten: Wladimir Iljitsch Lenin (aus seiner Schrift »Was tun?«). Es illustriert innerhalb seiner Pressetheorie eine der drei Funktionen der sozialistischen Parteipresse, nämlich die »Organisation« (neben der »Propaganda« und der »Agitation«).

Lenin befürwortete eine strikte Unterordnung der Parteipresse unter die Parteiführung und deren politische Linie. Nach der Oktoberrevolution zerstörte er die demokratische Öffentlichkeit in Russland durch die Unterdrückung Andersdenkender und das Verbot der gesamten nicht-bolschewistischen Presse (vgl. Poerschke 2020). Diese scheinbare geistige Nachbarschaft erklärt womöglich, dass die Idee des Konstruktiven Journalismus vielen Journalist*innen in westlichen De- 
mokratien (und auch in postsozialistischen Gesellschaften Osteuropas, wie ich bei Vorträgen vor russischen, ukrainischen und moldawischen Medienmacher*innen erlebte) Bauchschmerzen bereitet. Im Umkehrschluss liefert sie auch eine Begründung, warum so manche Führer postkolonialer Staaten in Afrika, die zuvor als Journalisten gearbeitet hatten und mit dem Marxismus-Leninismus und der Sowjetunion sympathisierten, von der Idee eines regierungsnahen Development Journalism angetan waren - etwa Kwame Nkrumah in Ghana, Jomo Kenyatta in Kenia und Julius Nyerere in Tansania (vgl. Xu 2009, 359).

Diesen Geist trägt jedoch weder die Idee des Konstruktiven noch des Transformativen Journalismus; er passt auch nicht zur Idee des WBGU einer Großen Transformation zur Nachhaltigkeit: Jener wünschenswerte Umbau von Werten, Praktiken und Institutionen soll nicht nach einem Masterplan von oben oktroyiert werden, sondern ein "gesamtgesellschaftlicher Suchprozess « sein, an dem »alle gesellschaftlichen Akteure [...] zu beteiligen sind « (WBGU 2011, 380), und der auf »Einsicht in die Notwendigkeit« (ebd., 341) setzt. Entsprechend bleibt ein Transformativer Journalismus, der hier (analog zu einer Transformativen Bildung und einer Transformativen Wissenschaft) als ein Berichterstattungsmuster unter mehreren konzeptualisiert wurde, ein autonomer Akteur, der zentrale journalistische Qualitätskriterien wie Unabhängigkeit, Kritik und Objektivität erfüllen soll. Er ist alles andere als wertneutral, sondern ausdrücklich wertgebunden - was allerdings kein Alleinstellungsmerkmal dieses Berichterstattungsmusters darstellt. So ist im westlichen Journalismus unumstritten, dass Journalist*innen die Demokratie verteidigen sollen, also an demokratische Werte (zu denen die Presse- und Meinungsfreiheit gehört) gebunden sind. Transformativer Journalismus bekennt sich darüber hinaus zum Schutz der natürlichen Lebensgrundlagen, ohne die Demokratie oder eine funktionierende Gesellschaft allgemein nicht möglich sind, ${ }^{10}$ und zu einer Reihe anderer Prinzipien, die im weiteren Sinn mit sozialer und politischer Gerechtigkeit zu tun haben (vgl. Wright 2017, 53) und unter dem Schirmbegriff »Nachhaltigkeit« von der Staatengemeinschaft weitgehend konsentiert sind.

10 Zu Recht fragt Schäfer $(2018,84)$, ob man sich als Journalist*in im Zeitalter des Anthropozäns zu ökologischer Nachhaltigkeit »auf berufsethischer Ebene überhaupt neutral verhalten kann« und ob Nachhaltigkeit »nicht mittlerweile zu einem universellen Wert geworden ist [...]. Ganz so wie Gleichberechtigung, freie Meinungsäußerung oder Minderheitenschutz, die interessanterweise weniger oft Thema für journalistische Selbstbefindlichkeiten sind.« 
Was in diesem Beitrag aus Platzgründen nicht geleistet werden konnte, ist ein Bezug der Idee des Transformativen Journalismus auf Transformationstheorien und -modelle. Anbieten würde sich der neomarxistische Ansatz von Wright (2017), der mögliche Wege in eine nachkapitalistische Gesellschaft per »Transformation durch Bruch«, »Transformation durch Freiräume« und »Symbiotische Transformation« sieht. Die reale Praxis des Konstruktiven und Transformativen Journalismus in der gegenwärtigen Gesellschaft ist demnach am ehesten mit der »Transformation durch Freiräume« verbunden: So wie der Kapitalismus einst in den Freiräumen der Feudalgesellschaft entstand, könne das gegenwärtige Wirtschaftssystem transformiert werden durch »Institutionen, die die angestrebte alternative Welt bereits im Hier und Jetzt verkörpern« (ebd., 441). Solche Institutionen, Praktiken und Akteur*innen werden von Transformativen Journalist*innen publizistisch begleitet und durch die Herstellung von Öffentlichkeit gefördert.

Denkbar ist ebenso eine theoretische Fundierung des Transformativen Journalismus mit Hilfe der »Multilevel Perspective on Transitions«. MLP ist eine Theorie mittlerer Reichweite, die ihre Wurzeln in den Science and Technology Studies, der Evolutionsökonomik, der Strukturationstheorie und dem Neo-Institutionalismus hat und erklärt, wie sich Innovationen aus Nischen in bestehenden soziotechnischen Regimen durchsetzen (Geels und Schot 2010). Im Modell der MLP wäre der Transformative Journalismus der Beobachter der Nischen, in denen öko-soziale Innovationen entwickelt werden - während der Mainstream-Journalismus, der auf das Abbilden des Elitendiskurses per »Objective Reporting « fokussiert ist, als Beobachter der etablierten Regime vorgestellt werden kann. Genaueres, auch bezüglich der Implikationen für das journalistische Rollenverständnis, die journalistische Ethik und die journalistische Qualität, bleibt einer späteren Ausarbeitung vorbehalten.

\section{Literatur}

Autorenkollektiv (verantwortlich für die Redaktion: Wolfgang Böttger). 1981. Wörterbuch der sozialistischen Journalistik. 2., wesentlich veränderte Auflage, KarlMarx-Universität Leipzig, Sektion Journalistik.

Beck, Ulrich. 2017. Die Metamorphose der Welt. Frankfurt am Main: Suhrkamp.

Beiler, Markus. 2013. Nachrichtensuche im Internet. Inhaltsanalyse zur journalistischen Qualität von Nachrichtensuchmaschinen. Konstanz: UVK.

Beiler, Markus, und Uwe Krüger. 2018. Mehr Mehrwert durch Konstruktiven Journa- 
lismus? Idee des Konzepts und Implikationen zur Steigerung des Public Values von Medien. In: Der öffentliche (Mehr-)Wert von Medien. Public Value aus Publikumssicht, herausgegeben von Nicole Gonser, 167-191. Wiesbaden: Springer VS.

Bendell, Jem. 2020. Deep Adaptation: A Map for Navigating Climate Tragedy. IFLAS Occasional Paper 2. http://lifeworth.com/deepadaptation.pdf. Zugegriffen: 8. Januar 2021.

Bennett, W. Lance. 1990. Toward a Theory of Press-State Relations in the United States. Journal of Communication 40 (2): 103-125.

Borner, Joachim, Anke Oxenfarth, und Manfred Ronzheimer. 2019. Impact-Journalismus und zielgenaues Storytelling für gesellschaftlichen Wandel. Sondierungsstudie des Umweltbundesamtes. Berlin: Kolleg für Management und Gestaltung nachhaltiger Entwicklung. http://kmgne.de/wp-content/ uploads/2019/12/Sondierungsstudie-des-Umweltbundesamtes-2019.pdf. Zugegriffen: 8. Januar 2021.

Bundesregierung. 2018. Deutsche Nachhaltigkeitsstrategie. https://www.bundesregierung.de/resource/blob/975274/1546450/65089964ed4a2ab07 ca8a4919e09e0af/2018-11-07-aktualisierung-dns-2018-data.pdf. Zugegriffen: 8. Januar 2021.

Buschow, Christopher. 2012. Strategische Institutionalisierung durch Medienorganisationen. Der Fall des Leistungsschutzrechtes. Köln: Herbert von Halem.

Cenite, Mark, Chong Shing Yee, Han Teck Juan, Lim Le Quin, und Tan Xian Lin. 2008. Perpetual development journalism? Balance and framing in the 2006 Singapore election coverage. Asian Journal of Communication 18 (3): 280-295.

Chalkley, Alan. 1969. A Manual of Development Journalism (Or how to help one thousand million Asians earn a decent living ... yes, we mean YOU). Cardiff: Thomson Foundation Editorial Study Centre und Press Foundation of Asia.

Donges, Patrick. 2006. Medien als Institutionen und ihre Auswirkungen auf Organisationen. Perspektiven des soziologischen Neo-Institutionalismus für die Kommunikationswissenschaft. Medien \& Kommunikationswissenschaft 54 (4): 563-578.

Dörre, Klaus, Stephan Lessenich, und Hartmut Rosa. 2017. Soziologie - Kapitalismus - Kritik. Eine Debatte. 5. Auflage. Frankfurt am Main: Suhrkamp.

Entman, Robert. 1993. Framing. Toward clarification of a fractured paradigm. Journal of Communication 43 (4): 51-58.

Galtung, Johan, und Mari Holmboe Ruge. 1965. The structure of foreign news. The presentation of the Congo, Cuba and Cyprus crises in four Norwegian newspapers. Journal of Peace Research 2 (1): 64-91.

Geels, Frank W., und Johan Schot. 2010. The Dynamics of Transitions: A SocioTechnical Perspective. In: Transitions to Sustainable Development. New Directions in the Study of Long Term Transformative Change, herausgegeben von John Grin, Jan Rotmans und Johan Schot, 11-104. New York, Abingdon: Routledge.

Göpel, Maja. 2016. The Great Mindshift. How a New Economic Paradigm and Sustainability Transformations go Hand in Hand. Wuppertal Institut und Springer Open. https://www.springer.com/de/book/9783319437651. Zugegriffen: 8. Januar 2021. 
Gyldensted, Cathrine. 2015. From mirrors to movers. Five elements of positive psychology in constructive journalism. Charleston: Group Publishing.

Haagerup, Ulrik. 2015. Constructive News. Warum »bad news« die Medien zerstören und wie Journalisten mit einem völlig neuen Ansatz wieder Menschen berühren. Salzburg: Oberauer.

Hamidi, Kefa, und Antonia Mielke Möglich. 2019. Entwicklungskommunikation Communication for Social Change. Aufbau und Etablierung eines neuen Schwerpunktes in der deutschen Kommunikationswissenschaft. Global Media Journal (German Edition) 9 (1): 1-15. https://www.db-thueringen.de/servlets/ MCRFileNodeServlet/dbt_derivate_00044867/GMJ17_Hamidi_Mielke_final. pdf. Zugegriffen: 8. Januar 2021.

Hanusch, Folker, und Charu Uppal. 2015. Combining detached watchdog journalism with development ideals: An exploration of Fijian journalism culture. The International Communication Gazette 77 (6): 557-576.

Herman, Edward S., und Noam Chomsky. 2002. Manufacturing Consent. The Political Economy of the Mass Media. New York: Pantheon.

Jarren, Otfried. 2015. Journalismus - unverzichtbar?! Publizistik 60 (2): 113-122.

Kannengießer, Sigrid. 2020. Nachhaltigkeit und das »gute Leben«. Zur Verantwortung der Kommunikations- und Medienwissenschaft in digitalen Gesellschaften. Publizistik 65 (1): 7-20.

Knoche, Manfred. 2002. Kommunikationswissenschaftliche Medienökonomie als Kritik der Politischen Ökonomie der Medien. In: Medienökonomie in der Kommunikationswissenschaft: Bedeutung, Grundfragen und Entwicklungsperspektiven. Manfred Knoche zum 60. Geburtstag, herausgegeben von Gabriele Siegert, 101-109. Münster: Lit.

Kramp, Leif, und Stephan Weichert. 2020. Nachrichten mit Perspektive. Lösungsorientierter und konstruktiver Journalismus in Deutschland. Frankfurt am Main: Otto-Brenner-Stiftung. https://www.otto-brenner-stiftung.de/fileadmin/user_ data/stiftung/02_Wissenschaftsportal/03_Publikationen/AH101_konstr_Journalismus.pdf. Zugegriffen: 8. Januar 2021.

Krüger, Uwe. 2018. Der neue Strukturwandel der Öffentlichkeit und die German Angst. In: Die neue Öffentlichkeit. Wie Bots, Bürger und Big Data den Journalismus verändern, herausgegeben von Gabriele Hoofacker, Wolfgang Kenntemich und Uwe Kulisch, 9-25. Wiesbaden: Springer VS.

Krüger, Uwe. 2019. Meinungsmacht. Der Einfluss von Eliten auf Leitmedien und Alpha-Journalisten - eine kritische Netzwerkanalyse. 2., überarbeitete und erweiterte Auflage. Köln: Herbert von Halem.

Krüger, Uwe. 2016. Mainstream - Warum wir den Medien nicht mehr trauen. 2., durchgesehene und aktualisierte Auflage. München: C. H. Beck.

Krüger, Uwe, und Nicole Gassner. 2014. Abschied von den Bad News. Message o. Jg. (1): 20-25. https://www.message-online.com/wp-content/uploads/SoJ.pdf. Zugegriffen: 8. Januar 2021.

Krüger, Uwe, und Michael Meyen. 2018. Auf dem Weg in die Postwachstumsgesellschaft. Plädoyer für eine transformative Kommunikationswissenschaft. Publizistik 63 (3): 341-357. 
Krüger, Uwe, und Juliane Pfeiffer. 2020. Die Neoklassische Ökonomik und der Romantische Konsumismus: Ideologische Bremsklötze einer »Großen Transformation« zur Nachhaltigkeit. In: Ideologie, Kritik, Öffentlichkeit: Verhandlungen des Netzwerks Kritische Kommunikationswissenschaft, herausgegeben von Uwe Krüger und Sebastian Sevignani, 212-239. Frankfurt am Main: Westend sowie Universität Leipzig. DOI: 10.36730/ideologiekritik.2019.10.

Loosen, Wiebke, Julius Reimer, und Sascha Hölig. 2020. Was Journalisten sollen und wollen: (In-)Kongruenzen zwischen journalistischem Rollenselbstverständnis und Publikumserwartung. Hamburg: Hans-Bredow-Institut. https://www.hansbredow-institut.de/uploads/media/default/cms/media/vhj8v7a_ AP49Was\%20Journalisten\%20wollen\%20und\%20sollen.pdf. Zugegriffen: 8. Januar 2021.

McIntyre, Karen. 2019. Solutions journalism: The Effects of Including Solution Information in News Stories about Social Problems. Journalism Practice 13 (1): $16-34$.

McIntyre, Karen, und Cathrine Gyldensted. 2017. Constructive Journalism: An Introduction and Practical Guide for Applying Positive Psychology Techniques to News Production. The Journal of Media Innovations 4 (2): 20-34.

Meier, Klaus. 2018. Journalistik. 4. Auflage. Konstanz: UTB.

Meier, Klaus. 2018. Wie wirkt Konstruktiver Journalismus? Ein neues Berichterstattungsmuster auf dem Prüfstand. Journalistik 1 (1): 4-25. https://journalistik.online/ausgabe-012018/wie-wirkt-konstruktiver-journalismus/. Zugegriffen: 8. Januar 2021.

Michelsen, Gerd. 2007. Nachhaltigkeitskommunikation: Verständnis - Entwicklungen - Perspektiven. In: Handbuch Nachhaltigkeitskommunikation. Grundlagen und Praxis, herausgegeben von Gerd Michelsen und Jasmin Godemann, 2. Auflage, 25-41. München: Oekom.

Michelsen, Gerd, und Daniel Fischer. 2016. Vom Umwelt- zum Nachhaltigkeitsjournalismus? Die Initiative »Nachhaltigkeit und Journalismus«. In: Nachhaltigkeit und Journalismus. Erkenntnisse und Impulse aus Wissenschaft und Praxis, herausgegeben von Gerd Michelsen und Daniel Fischer, 9-19. Bad Homburg: Verlag für Akademische Schriften.

Neverla, Irene. 2020. Nachhaltigkeit: Zur Theoretisierung einer politischen Kategorie. Publizistik 65 (3): 333-339.

Poerschke, Hans. 2020. Das Prinzip der Parteiliteratur. Partei und Presse bei und unter Lenin 1899-1924. Köln: Herbert von Halem.

Polanyi, Karl. 1957. The Great Transformation: The Political and Economic Origins of Our Time. Boston: Beacon Press.

Pöttker, Horst. 2003. Kommunikationsstörungen? Zur Systematik der sozialen Beziehung zwischen Politikern und Journalisten. In: Machtdarstellung und Darstellungsmacht. Beiträge zur Theorie und Praxis moderner Politikvermittlung, herausgegeben von Ulrich Sarcinelli und Jens Tenscher, 149-167. Baden-Baden: Nomos.

Ronzheimer, Manfred. 2013. Vom Nullthema in den Mainstream. Die Rolle der Medien in der Transformation. Politische Ökologie 133 (31): 118-123. https:// 
jbzzukunftsbuch.files.wordpress.com/2014/12/politische-c3b6kologie-transformationsjournalismus.pdf. Zugegriffen: 8. Januar 2021.

Schäfer, Torsten. 2018. Geschichten als Chance. Erzählformen im Umweltjournalismus. In: Politische Ökologie 152: 83-90. München: Oekom.

Schmidt, Jasmina. 2017. Konstruktiver Journalismus - ein Ansatz zur kosmopolitischen Vermittlung fernen Leids? Global Media Journal (German Edition) 7 (2): 1-26. https://www.db-thueringen.de/servlets/MCRFileNodeServlet/dbt_derivate_00039841/GMJ14_Schmidt_final.pdf. Zugegriffen: 8. Januar 2021.

Schneidewind, Uwe. 2018. Die Große Transformation. Eine Einführung in die Kunst gesellschaftlichen Wandels. Frankfurt am Main: S. Fischer.

Schultz, Tanjev. 2020. Der Reporter-Blick von nirgendwo? Journalismus in der Spannung zwischen Objektivität und Subjektivität. Publizistik. DOI: https:// doi.org/10.1007/s11616-020-00624-1. Zugegriffen: 8. Januar 2021.

Scott, Martin, Mel Bunce, und Kate Wright. 2017. Donor Power and the News: The Influence of Foundation Funding on International Public Service Journalism. The International Journal of Press/Politics 22 (2): 163-184.

Sevignani, Sebastian. 2016. Kritische Politische Ökonomie. In: Handbuch Medienökonomie, herausgegeben von Jan Krone und Tassilo Pellegrini, 1-28. Wiesbaden: Springer VS.

Urner, Maren. 2019. Schluss mit dem täglichen Weltuntergang. Wie wir uns gegen die digitale Vermüllung unserer Gehirne wehren. München: Droemer Knaur.

Waisbord, Silvio. 2010. Rethinking »Development« Journalism. In: The Routledge Companion to News and Journalism, herausgegeben von Stuart Allan, 148-158. London: Routledge.

WBGU. 2011. Welt im Wandel. Gesellschaftsvertrag für eine Große Transformation. Hauptgutachten des Wissenschaftlichen Beirats der Bundesregierung Globale Umweltveränderungen. Bonn. https://www.wbgu.de/fileadmin/user_upload/wbgu/publikationen/hauptgutachten/hg2011/pdf/wbgu_jg2011.pdf. Zugegriffen: 8. Januar 2021.

Welzer, Harald. 2013. Selbst denken. Eine Anleitung zum Widerstand. Frankfurt am Main: S. Fischer.

Wright, Erik Olin. 2017. Reale Utopien. Wege aus dem Kapitalismus. Frankfurt am Main: Suhrkamp.

Xu, Xiaoge. 2009. Development Journalism. In: The Handbook of Journalism Studies, herausgegeben von Karin Wahl-Jorgensen und Thomas Hanitzsch, 357370. New York: Routledge.

\section{Open Access}

Dieser Beitrag erscheint unter der Creative-Commons-Lizenz CC-BY-ND 3.0 DE: https://creativecommons.org/licenses/by-nd/3.0/de/. 